\title{
Analogies between dark solitons in atomic Bose-Einstein condensates and optical systems
}

\author{
N.P. Proukakis ${ }^{\dagger}$, N.G. Parker ${ }^{\dagger}$, D.J. Frantzeskakis ${ }^{\ddagger}$, and \\ C.S. Adams ${ }^{\dagger}$ \\ $\dagger$ Department of Physics, University of Durham, South Road, Durham DH1 \\ 3LE, United Kingdom \\ $¥$ Department of Physics, University of Athens, Panepistimiopolis, Zografos, \\ Athens 15784, Greece \\ E-mail: n.p.proukakis@durham.ac.uk
}

\begin{abstract}
.
Dark solitons have been observed in optical systems (optical fibers, dielectric guides and bulk media), and, more recently, in harmonically confined atomic BoseEinstein condensates. This paper presents an overview of some of the common features and analogies experienced by these two intrinsically nonlinear systems, with emphasis on the stability of dark solitons in such systems and their decay via emission of radiation. The closely related issue of vortex dynamics in such systems is also briefly discussed.
\end{abstract}

PACS numbers: $03.75 . \mathrm{Lm}, 42.65 . \mathrm{Tg}, 42.81 . \mathrm{Dp}$ 


\section{Introduction}

Nonlinear systems in physics support the appearance of solitary waves [1, which propagate without dispersion. Such waves have been extensively studied, both theoretically and experimentally in diverse systems, such as shallow water waves [2], macromolecules [3], acoustics 4], plasma physics [5], elastic surfaces [6], optical fibers [7], condensed excitons in crystals [8] and, more recently, realised experimentally in atomic Bose-Einstein condensates (BECs) 9] 10. Solitary waves are of two kinds, depending on the sign of the effective nonlinearity, whose physical origin and interpretation is system-dependent. If the effective nonlinearity is attractive, then bright solitons are formed, whereas dark solitons arise in the opposite case (e.g. defocusing media). Bright solitons are non-dispersive (positive) density waves, whereas dark solitons correspond to density depressions characterised by a phase shift across their density minimum. The behaviour of these two distinct non-dispersive excitations is very different.

This paper focuses on the case of dark solitons. Dark solitons have been investigated at length in nonlinear optics [11, where potential applications in optical communications and in photonics have also been proposed (see, e.g., relevant experimental results in [12, 13], and also [14]). This paper aims to highlight some common features between optical and atomic dark solitons [15], in the context of the dynamics of dark solitons in BECs. Although very different in origin, the underlying equation describing the dynamical properties of dark solitons in nonlinear optical systems and atomic BECs has the same structure, given by the following dimensionless Nonlinear Schroedinger Equation (NLSE) (usually referred to as Gross-Pitaevskii equation (GPE) in the context of BECs) [16

$$
i \frac{\partial \psi}{\partial t}=-\frac{1}{2} \nabla_{d}^{2} \psi+F\left(|\psi|^{2}\right) \psi+V_{\mathrm{ext}} \psi
$$

In nonlinear optics, $\psi$ is the (complex) electric field envelope, the variable $t$ plays the role of the propagation distance $z$ (along the waveguide or fiber), while the righthand side has a different sense for temporal solitons (in optical fibers) or spatial solitons (in bulk media or dielectric waveguides). In the case of temporal solitons, $d=1$ and $\nabla_{1}^{2} \psi=\partial^{2} \psi / \partial \tau^{2}$ describes the normal dispersion (where $\tau$ is a retarded time measured in a frame of reference moving with the group velocity), while, in the case of spatial solitons, $\nabla_{d}^{2} \psi$ describes the beam's diffraction (here $d=2$ and $\nabla_{2}^{2}$ is the transverse Laplacian). On the other hand, $F\left(|\psi|^{2}\right)$ is proportional to the intensity-dependent change of the refractive index of the optical medium $\left(I=|\psi|^{2}\right.$ is the light intensity). In optical fibers, the nonlinearity is of the Kerr type, i.e., $F\left(|\psi|^{2}\right)=|\psi|^{2}$. In bulk nonlinear media (such as vapors, semiconductors, polymers, etc), the nonlinearity may be of a non-Kerr type, e.g., a competing, or, generally, a saturable nonlinearity [11], which, in some cases (e.g., relatively low light-intensities), can be approximated by the Kerr nonlinearity. Finally, in the case of dark spatial solitons in dielectric waveguides, the term $V_{\text {ext }}$ accounts for a possible change of the linear part of the refractive index in the transverse dimensions. As an example, in an inhomogeneous nonlinear waveguide, the refractive index $n_{w}$ may take the form $n_{w}=n_{0}-n_{1}\left(x^{2}+y^{2}\right)-n_{2}|\psi|^{2}$, where $n_{j}>0(j=0,1,2)$ represent the homogeneous, inhomogeneous and nonlinear (Kerr) parts, respectively (see, e.g., [17]). This case bears resemblance to the typical situation occuring in BECs, where the condensate is usually trapped in a parabolic magnetic potential [16] (see below). Notice that in the case of the nonlinear waveguide, the propagation distance $t$ in equation (1) is measured 
in units of the diffraction length $L_{D}=\beta_{0} w_{0}^{2}$ ( $\beta_{0}$ is the longitudinal wavenumber and $w_{0}=\left(2 \beta_{0}^{2} n_{1} / n_{0}\right)^{-1 / 4}$ is a transverse length scale), the transverse coordinates $x, y$ in units of $w_{0}$ and the electric field envelope in units of $\left(\beta_{0} L_{D} n_{2} / n_{0}\right)^{-1 / 2}$.

In the context of BECs, $\psi$ corresponds to the macroscopic order parameter of the system at zero temperature (or, in general. for temperatures much lower than the temperature at which the BEC phase transition occurs), and this can be thought of as the wavefunction describing the condensate. It is a complex parameter that can be expressed as $\psi=\sqrt{n} \exp (i \phi)$, where $n$ is the atomic density and $\phi$ its phase. The terms appearing on the right hand side of equation (1) denote, respectively, the kinetic energy contribution in a $d$-dimensional manifold (in the general case, $\nabla_{d}^{2}$ with $d=3$ is the transverse Laplacian in 3-dimensions), the strength and form of the nonlinearity and the external confinement of the system under consideration. It should be noted that in atomic BECs, the nonlinearity is also an intrinsic phenomenon, arising from the scattering properties between the atoms of the condensate. Nevertheless, for sufficiently dilute atomic gases, only s-wave two-body scattering is important, and, as a result the corresponding nonlinear term in equation (1) can usually be approximated as $F\left(|\psi|^{2}\right)=|\psi|^{2}$, resembling the Kerr nonlinearity term appearing in optics. The last term can generally account for any external confinement imposed on the medium in which soliton propagation may take place. Presence of such a spatially-dependent term (accounting for the graded-index waveguide in optics or the confining potential in BECs) modifies the local background density in the medium, which thus becomes non-uniform. Excluding spatially-dependent refractive indices, optical waveguides are generally homogeneous systems, whereas atomic BECs (unlike other superfluids, such as ${ }^{4} \mathrm{He}$ ) are typically formed in axially symmetric harmonic (magnetic) traps, featuring inhomogeneous confinement along all three of the system's directions, with $V_{\text {ext }}=(1 / 2) m\left(\omega_{z}^{2} z^{2}+\omega_{r}^{2}\left(x^{2}+y^{2}\right)\right)$, where $\omega_{z}\left(\omega_{r}\right)$ the longitudinal (transverse) confining frequency. This leads to very distinct dynamics between the two systems, since dark solitons in BECs propagate on a position- (and time-) dependent background, in stark contrast to the constant background in optical waveguides

This paper focuses on the case of a cubic nonlinearity, which is relevant to both optical and atomic systems. In this case, strictly speaking (and from a mathematical point of view), a NLSE dark soliton is a solution of equation (1) in one dimension (i.e. $d=1$, for which $\nabla_{1}^{2}=\left(\partial^{2} / \partial z^{2}\right)$ ), on a homogeneous background density (i.e. in the absence of the third term appearing above), which reduces to

$$
i \frac{\partial \psi}{\partial t}=-\frac{1}{2} \frac{\partial^{2}}{\partial z^{2}} \psi+|\psi|^{2} \psi
$$

In order to reduce the 3D Gross-Pitaevskii equation to the above 1D form, one requires (i) very tight transverse (radial) confinement, such that transverse excitations are completely suppressed, and (ii) a longitudinally homogeneous density $\left(\omega_{z}=0\right)$. In situations of tight radial confinement, one obtains an effective one-dimensional interaction strength $g$ by integrating the three dimensional interaction strength $g_{3 D}$ over the transverse directions. Since $g_{3 D}=4 \pi \hbar^{2} a / m$ (where $a$ is the $s$-wave scattering length characterising the atomic interactions and $m$ the atomic mass), this yields $g=g_{3 D} /\left(2 \pi l_{r}^{2}\right)$, where $l_{r}=\sqrt{\hbar / m \omega_{r}}$ is the transverse harmonic oscillator length. To further reduce the resulting equation to dimensionless form, length is scaled in units of the fluid healing length $\xi=\hbar / \sqrt{n_{0} g m}$, velocity in terms of the Bogoliubov speed of sound for the medium $c=\sqrt{n_{0} g / m}$, and the atomic density rescaled by the peak density $n_{0}$. Energy is scaled in terms of the chemical potential of the system $\mu=g n_{0}$. 
The wavefunction of a dark soliton propagating with speed $v$ and position $(z-v t)$ on a uniform background of unity is given analytically by [18

$$
\psi(z, t)=e^{-i t}(\lambda \tanh [\lambda(z-v t)]+i v) .
$$

Here $\lambda=\sqrt{1-(v / c)^{2}}$, and the healing length $\xi$ corresponds roughly to the size of the soliton. The soliton speed $v$ depends on its depth $n_{d}$ relative to the background density and the phase slip $S$ across its centre via $v / c=\sqrt{1-\left(n_{d} / n_{0}\right)}=\cos (S / 2)$.

The fact that dark solitons are characterized by a nontrivial distribution of their phase (actually, initially, i.e. at $t=0$, the soliton phase is an odd function of $x$ ), raised certain technical difficulties for the experimental verification of their propagation in optical systems. That is why the first experimental attempts to study dark temporal solitons in optical fibers took place in the late 1980's, even though dark solitons were predicted to occur in optical fibers as early as 1973 [19]. These first experiments reported the creation of a fundamental dark soliton [i.e., $v=0$ in equation (3)] (using a $\pi$-phase step) [20, the evolution of a pair of small-amplitude dark solitons (emerging from an even dark pulse) [21], as well as the generation and subsequent evolution of a dark soliton (emerging from a background pulse with a phase jump) 22]. Later, during the 1990's, the generation of dark soliton trains at high repetition rates [23], as well as their potential applications in optical communications [12] was demonstrated. Note that the first experimental results for the generation of spatial dark solitons (upon using proper amplitude and phase masks) were reported in sodium vapor [24], bulk semiconductors [25], photorefractive materials [26], etc., at around the same time.

On the other hand, matter-wave dark solitons have also been observed in several recent experiments [9]. Similarly to the case of the optical systems, a quantumphase engineering technique (or phase-imprinting method) was used to imprint the appropriate phase distribution on the BEC so as to create dark solitons. The phaseimprinting method (which was also employed for the creation of vortices in BECs [27. 28]) is an efficient tool to engineer the phase of the BEC clouds [29, 30, 31] and its optimization has been discussed in 32. In particular, in that work it was suggested that such an optimization involves engineering not only the phase, but also the BEC density, which may result in the generation of stationary dark matterwave solitons. Apart from the phase-engineering technique and the above mentioned optimized version of it (i.e., the so-called phase and density engineering technique [32]), Burger et al. 33 recently proposed the creation of dark solitons in BECs by purely engineering the density distribution. Such a "density engineering" technique bears resemblance to the creation of optical solitons by intensity modulations of a backround light field, as in relevant experiments in optical fibers (see, e.g., 21] and relevant theoretical work in 34).

The idealised soliton of equation (3) propagates in a stable manner. However, realistic conditions deviate from this idealised equation (equation (2)), thus breaking the integrability of the system and rendering the soliton unstable to decay via sound emission. There are different types of instabilities which can arise. Firstly, a dark soliton in usually embedded in higher than one-dimensional (1D) geometry. This leads to additional kinetic energy contributions in the transverse directions, which are expected to lead to the dominant decay mechanism, if the system is far from the 1D limit. Optical fibers are essentially 1D objects, thus eliminating, to large degree such instabilities. Initial experiments with condensates focused on 3D geometries. In lower dimensionalities, long wavelength excitations tend to destroy the universal phase coherence across the trapped atomic system (in accordance with the disappearance of 
BEC in dimensions $d \leq 2$ for a homogeneous system). However, it has recently been shown that, at ultralow temperatures, the universal coherence across the system is essentially restored [35, 36]. This regime is now a topic of active experimental and theoretical research. Quasi-one-dimensional atomic systems can be realised by the application of very tight transverse confinement [37, such that the transverse degrees of freedom become essentially frozen out [38. Such quasi-1D systems are particularly important in so-called atom chips [39, 40, 41, 42, where the motion is confined along only one direction by a current carrying wire. Hence, the domain of quasi-1D is now also accessible experimentally in atomic BECs. Experiments with dark solitons have so far been performed in 3D BECs [9], but one can now examine their dynamics in quasi-1D systems [43, in which their stability is expected to be largely enhanced [4].

A second source of instability arises from modifications of the above nonlinearity. In the general case of a non-cubic nonlinearity, equation (2) ceases to be a completely integrable system and as a result dark solitons are subject to perturbations that may be quite strong. This is particularly important, especially in the case of optical media, where deviations from the rather simple model of the Kerr nonlinearity may be significant. In such cases, more realistic models, such as the saturable nonlinearity, are relevant. Since the nonlinearity in atomic BECs arises from interactions within the condensate atoms, an additional dissipative mechanism will arise due to the presence of thermal (uncondensed) atoms in the medium. This effect is analogous to damping of the superfluid component in liquid helium, due to coupling with the normal component [45], and can be suppressed by going to extremely low temperatures in the atomic traps [4]. One should also mention that if the confinement becomes extremely tight, the nonlinearity in atomic BECs start deviating from the cubic form discussed throughout this work [47, and soliton propagation in this regime will be discussed elsewhere. Additional decay mechanisms arise from modifications from a homogeneous background, an important effect in atomic BECs. For completeness, one should also mention the effect of quantum fluctuations which have been shown to lead to decay in the case of deep slow solitons 48 .

Soliton decay due to the above instabilities is typically accompanied by the emission of sound waves. These sound waves are density waves in atomic condensates, and correspond to electromagnetic radiation in the optical context. Note, however, that, under appropriate conditions, the soliton may re-interact with the emitted sound, with the effect of stabilising it against decay. This occurs, for example, for dark solitons in quasi-1D condensates featuring longitudinal confinement, where stabilisation arises as a result of continuous sound emission and reabsorption cycles [49].

This paper is organised as follows: Section 2 discusses instabilities due to transverse excitations, whereas the rest of the paper focuses on the limit of tight transverse confinement, where this effect is negligible. In section 3 we highlight analogies between dark solitons propagating through potential steps and soliton transmission through regions of different indices of refraction. Section 4 outlines the dominant decay mechanisms in one-dimensional systems arising in both atomic and optical systems. A common result for the sound emission is found to be obeyed in this limit. In section 5, we briefly address the issue of stabilisation of dark solitons against decay mechanisms. Section 6 discusses analogous effects in vortices, a higher manifold topological structure which has recently been observed in both optical and atomic systems. Our concluding remarks are presented in section 7 . 


\section{Transverse Instabilities}

\subsection{Optical Dark Solitons}

In optical systems a tight transverse confinement of light beams can be realized in waveguide geometries (such as the optical fiber or the slab waveguide), in which the dark solitons are practically $1 \mathrm{D}$ objects, that are not prone to transverse instabilities. However, in planar waveguides or in bulk media, where the optical systems become effectively $2 \mathrm{D}$ or $3 \mathrm{D}$ respectively, the rectilinear dark solitons (i.e., the dark soliton stripes) are subject to a long-wavelength transverse instability (the so-called "snake instability"), which leads to the stripe breakup and the eventual creation of optical vortex solitons with alternate topological charges. The instability band is characterized by a maximum modulation wavenumber $Q_{c r}$ (depending on the dark-soliton amplitude), i.e., the dark soliton stripe is stable only for $Q>Q_{c r}$, where $Q$ is the perturbation wavenumber 50 .

In the early experiments [24, 51], where dark soliton stripes and grids were created on the transverse cross-section of an optical beam propagating through a bulk self defocusing medium (i.e., sodium vapor), the snake instability was not observed due to the finite-size of the beam and the weak nonlinearity of the medium. However, in later experiments in rubidium vapor [52] (relevant results have also been reported using photorefractive crystals [53]), the enhancement of the nonlinearity of the optical medium with increasing temperature, led to the direct experimental observation of the snake instability of a dark soliton stripe and the subsequent creation of vortex solitons (see also [54 for a review).

\subsection{Dark Solitons in Atomic BECs}

The snake instability arises in atomic systems as well; there, recent experiments have demonstrated the decay of the rectilinear dark solitons into vortex rings [55]. As in the case of nonlinear optics, this decay mechanism dominates far from 1D geometries, with the longitudinal sound emission being negligible in comparison. The effect of the snake instability was studied theoretically in a series of works [56, 57 and it has been suggested that it can be suppressed by a sufficiently strong transverse confinement of the condensate [4], which bears resemblance to the use of finite-size beams in nonlinear optics. Another possibility for the suppression of the snake instability is to bend a dark soliton stripe to form a ring of length $L<2 \pi / Q_{c r}$. Such "ring dark solitons" were predicted theoretically [58] and observed experimentally [59] in the context of optics. Recently, ring dark solitons were predicted to occur in BECs as well [60, with the snake instability leading to robust vortex arrays in the form of necklaces. Suppression of the snake instability in BEC experiments can be achieved, for example, in highly-elongated ("cigar-shaped") geometries. Here we aim to study the mechanism of the snake instability in BECs, upon considering a cylindricallysymmetric 3D geometry, explicitly featuring longitudinal harmonic confinement.

The transverse stability of a dark soliton in a harmonic trap can be studied by monitoring the evolution of a stationary dark soliton created at an off-center position. This is based on numerical simulations of the cylindrically-symmetric GPE. The initial density and phase profiles in the $z-r$ plane are shown in figure 1 , (where $z$ is the longitudinal and $r$ the transverse direction). The phase profile (b) features a steplike $\pi$ phase slip across the soliton minimum. Due to the inhomogeneous background density, the soliton accelerates towards the center, with its motion being subject to 

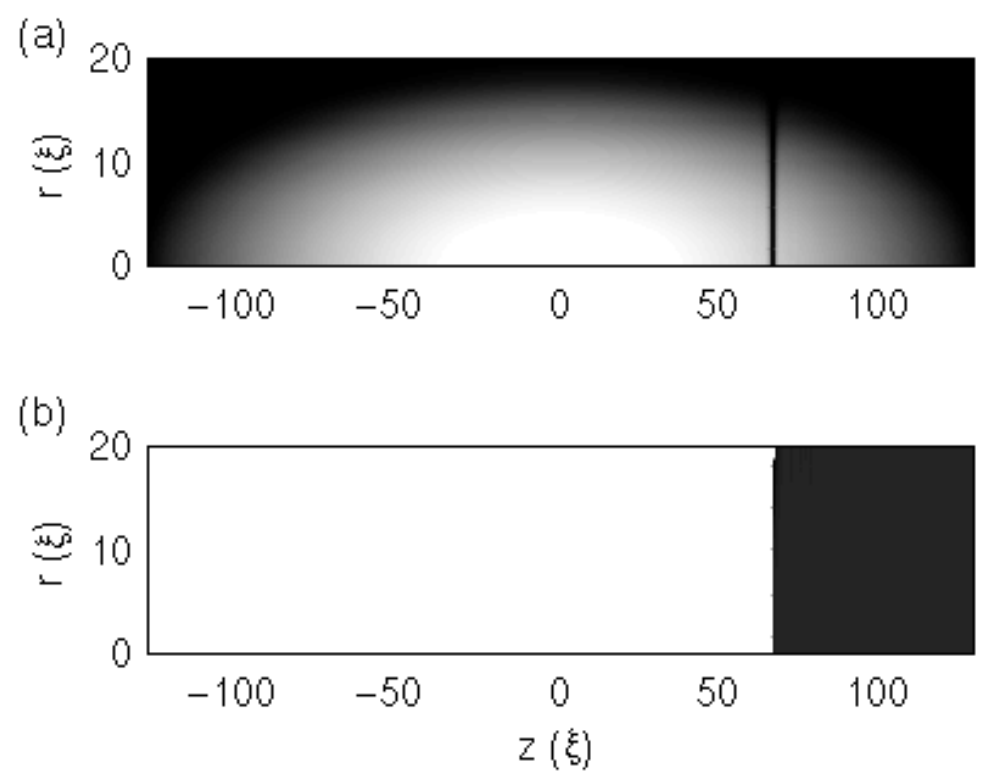

Figure 1. Planar view of (a) density and (b) phase profiles of a stationary off-centred dark soliton in an atomic Bose-Einstein condensate confined in a cylindrically-symmetric 3D trap. Here $\mathrm{z}$ denotes the longitudinal and $\mathrm{r}$ the transverse direction. The dark soliton, positioned at $z=66 \xi$, appears as a notch of zero density in the z-r plane and clearly features an abrupt phase slip of $\pi$. The trap frequencies are $\omega_{z}=\sqrt{2} \times 10^{-2}(\mu / \hbar)$ and $\omega_{r}=0.1(\mu / \hbar)$, giving a trap ratio $\omega_{r} / \omega_{z} \sim 7.1$. The chemical potential of the system, $\mu$, in terms of which energies are scaled, is obtained, by fixing the peak $3 \mathrm{D}$ density of the system to 1 . Note that, in the density (phase) scale, white represents the peak density $(\pi / 2)$ and black represents zero $(-\pi / 2)$.

strong coupling between the longitudinal and transverse degrees of freedom. Since the soliton is not the lowest energy state in $3 \mathrm{D}$ geometries, it tends to decay into more stable, lower energy structures, such as vortex rings. This occurs due to the existence of a mode with imaginary frequency components [56 [57. To probe this decay mechanism, figure 2 shows successive (a) density and (b) phase snapshots of a soliton in a three-dimensional geometry, focusing on the interesting regions around the soliton minimum. One clearly observes the anticipated snake-like bending of the soliton plane, followed by the decay into vortex rings and sound waves (the latter not easily visible on the greyscale used in this subsection, as their density is typically much less than that of the vortex rings). Cross-sections of these rings in the $z-r$ plane can be seen in (iv), with each vortex ring indicated by an arrow.

Such a decay mechanism involving the bending of the soliton plane will clearly tend to be suppressed as the transverse size of the condensate decreases, leading eventually to its complete prevention in highly-elongated quasi-1D geometries. The regimes of transverse stability of a moving soliton have been studied in 61. To highlight the effect of transverse confinement on soliton stability, figure 3 shows density and phase profiles of the evolution of an initially off-centred stationary soliton at the time when the soliton (or its decay products) have reached the trap centre, in traps of different transverse confinement. The top figure corresponds to the unstable $3 \mathrm{D}$ case 


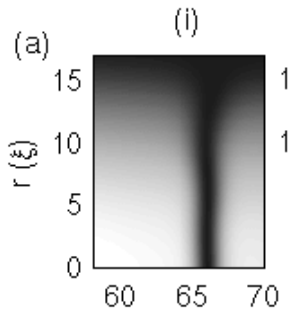

(ii)



(iii)

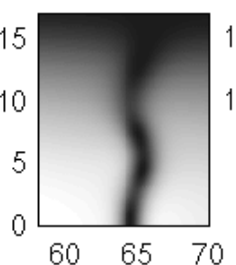

(iv)

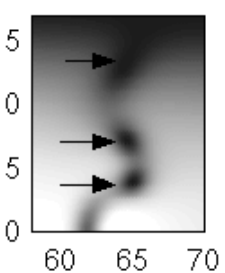

(b)
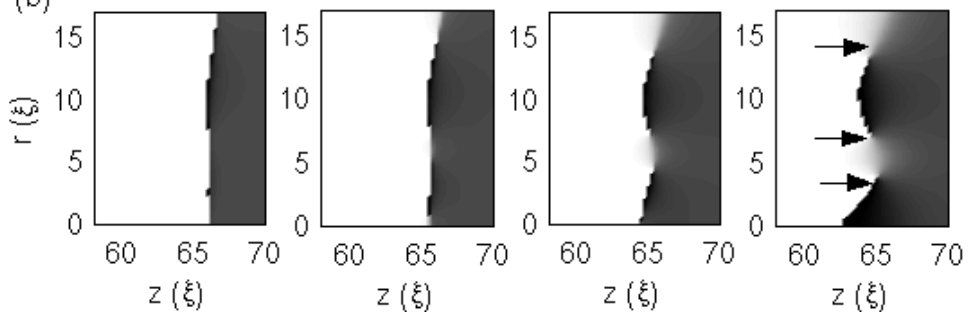

Figure 2. Close-up snapshots of the evolution of (a) density and (b) phase profiles for the initial soliton configuration of figure 1, depicting the main stages in the dynamics of the snake instability. Successive images correspond to $t \approx(\mathrm{i})$ 15, (ii) 19, (iii) 23 and (iv) $27 \xi / c$. In (iv), the dark soliton has already decayed into 3 vortex rings, as evident by the corresponding phase profile. The location of these vortex rings is indicated by arrows.

examined in figures 1 and 2 . In this case, the $2 \pi$ vortex phase singularity exhibited by the central vortex ring is evident in figure 3(b)(i), having been highlighted by a hollow white circle. Tightening the transverse confinement leads to a decrease in the bending of the soliton, and hence the production of less vortex rings, an issue studied in detail in 62. Figure 3(a)(ii) shows the case of a single vortex ring being produced, with the corresponding phase profile labelled in figure 3(b)(ii). Finally, very tight transverse confinement, leads to an effectively 1D system, featuring a stable soliton, as evident from the step-like phase profile of figure 3(b)(iii).

Lifetimes of dark solitons are hence clearly enhanced in geometries featuring tight transverse confinement, for which the chemical potential is too small to allow for transverse modes to be excited due to atom-atom interactions (or thermal effects). Any further studies of dark soliton dynamics will therefore be performed in highly elongated geometries, where the above decay mechanism is largely suppressed, and this will be assumed in all subsequent sections. All following results are therefore obtained by solving the 1D GPE with a suitable potential $V(z)$.

\section{Propagation through regions of different potential energy}

\subsection{Optical Solitons}

One interesting application of nonlinear optics is in nonlinear waveguides, which have been proposed as all-optical devices that may be used as switchers, modulators, bistable-optical elements, etc. An important effect in this context is the selflocalization of optical beams, which leads to the formation of finite-size self-focused 

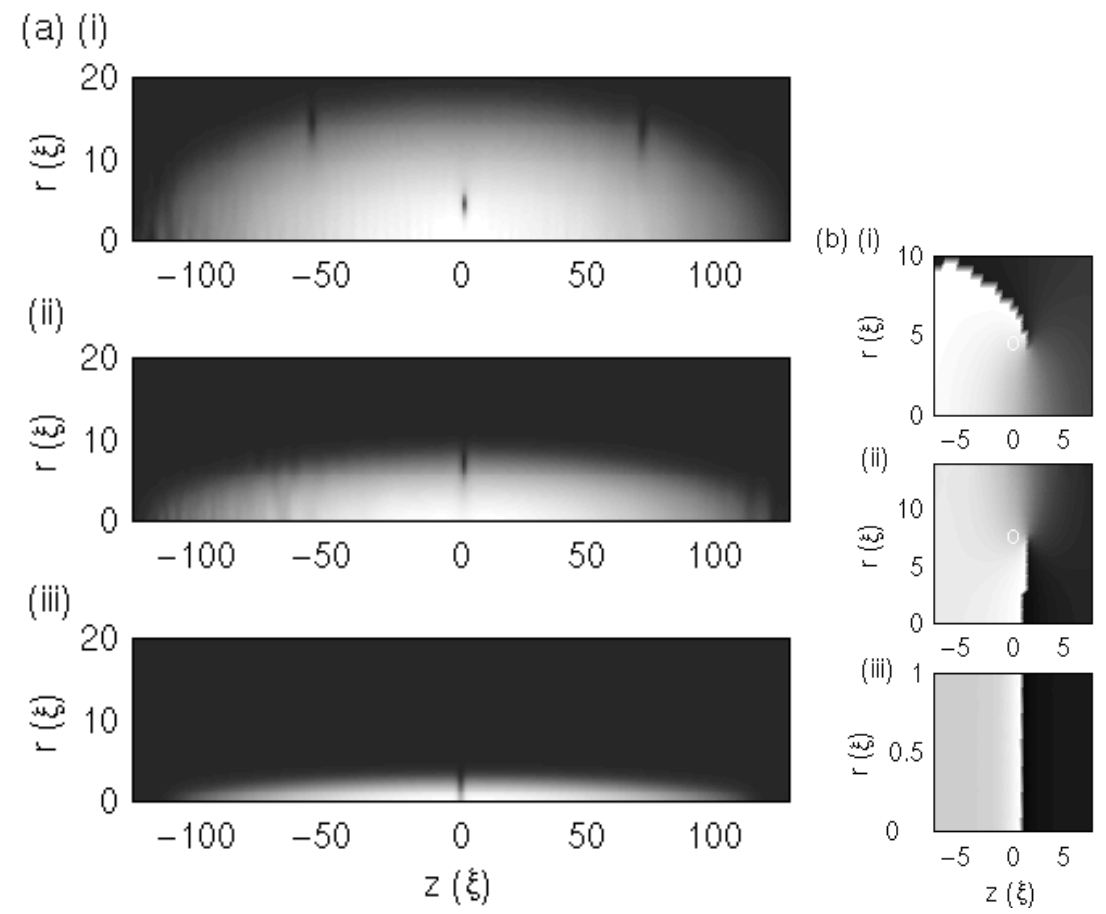

Figure 3. (a) Density (left) and (b) phase (right) profiles of the evolution of an initially off-centred stationary dark soliton for different transverse confinement. All simulations start with a dark soliton located at $z=66 \xi$, with the images shown at $t=$ (i) 279 , (ii) 193 , and (iii) $160 \xi / c$, after the soliton creation. From top to bottom, the aspect ratio of transverse to longitudinal harmonic confinement is $\left(\omega_{r} / \omega_{z}\right)=\left(\right.$ i) 7.1 , (ii) 14.2 , and (iii) 35.5 , corresponding to $\left(\mu / \hbar \omega_{r}\right)=($ i) 10 , (ii) 5 , (iii) 2, for the employed longitudinal trap frequency $\omega_{z}=\sqrt{2} \times 10^{-2}(\mu / \hbar)$. (i) Dark soliton has already decayed into 3 vortex rings, with the $2 \pi$ phase singularity of the central vortex ring shown in (b)(i), and highlighted by the hollow white circle. (ii) Soliton decays into a single vortex ring (phase profile on the right). (iii) Soliton in very elongated quasi-1D condensate is stable against the snake instability, as shown by the step-like $\pi$ phase difference across it.

channels, as well as to novel stationary nonlinear guides and surface waves in thin-film planar waveguides and at dielectric interfaces (see, e.g., 63]).

Considering that the self-focused light channels can be well-approximated by spatial bright solitons, it has been shown 64 that their dynamics near interfaces separating linear and nonlinear media or different nonlinear media, can be reduced to the study of motion of an equivalent particle in an effective step-like potential. This so-called "equivalent particle theory actually" corresponds to the adiabatic (leadingorder) approximation of the perturbation theory of solitons [65], while a higher-order approximations allows for inclusion of radiation effects following the beam reflection 66]. Adopting this approach, the propagation of light beams in planar thin-film nonlinear waveguides has been studied in detail 67 .

To link the above discussion with the following one referring to BECs, it is important to stress that in all the above works, as well as in earlier relevant contributions 68, 69, the scattering of an optical beam by an interface separating 
two media of different refractive indices can effectively be described by the dynamics of a NLSE soliton in a step-like potential.

\subsection{Solitons in Atomic BECs}

The analogous situation in the case of dark solitons in BECs is propagation into a region of different potential energy. This situation can be achieved by the addition of a finite size potential step along the condensate long axis, leading to a change in the local background density over a finite length, a situation illustrated schematically in figure 4(a). For sufficient wide steps, as employed here, the density in the step region is reduced to the Thomas-Fermi value, $n(z)=n_{0}-V(z)$. The behaviour of the soliton (figure 4(b)) depends crucially on whether the potential step is positive or negative. In the case of a lower potential energy region, i.e. higher density (dashed line), the soliton transmits through the intermediate region. Upon striking each interface, the soliton emits two counter-propagating sound pulses, as shown in figure 4(c)(i). A higher density in the step region leads to a higher speed of sound. If the step height is small and positive (dotted line in figure 4(b), figure 4(c)(ii)), then the situation is similar, with the main difference being that the speed of sound on the step is reduced. However, when the step height exceeds a critical limit, the soliton is reflected (possibly after spending a long time on the interface) (solid line in figure 4(b), figure 4(c)(iii)). To first order, the soliton, with depth $\left(n_{0}-v^{2}\right)$, will be reflected when the density at the step becomes too shallow to support it, i.e. when $V_{0}>v^{2}$ (assuming the density at the step heals to the Thomas-Fermi value). In actual fact, the soliton becomes reflected at a slightly higher step height due to the effects of sound emission, which causes a small decrease in the soliton depth. This effect is described in detail in [70]. Further increases in step height tend to restrict the sound emission, and in the limit of collision with a hard wall, $V_{0} \gg \mu$, the soliton reflects elastically. The loss in the soliton energy, caused by the sound emission leads to an ultimate increase in the soliton speed. The difference between initial and final soliton speeds is illustrated in figure 5 , for both cases of higher and lower potential steps.

It should be noted that a qualitatively similar scattering behavior of dark solitons has also been found using the equivalent particle theory in the limiting case where the potential step becomes a localized repulsive impurity [71].

\section{Longitudinal Instabilities}

\subsection{Optical Dark Solitons}

As mentioned in the Introduction, optical dark solitons formed in quasi-1D homogeneous geometries (i.e., temporal ones in optical fibers or spatial ones in weakly nonlinear bulk media and dielectric waveguides) are not prone to transverse perturbations (i.e., to the snake instability). As a result, propagation based on equation (1) (with $d=1$ ) will be stable. However, especially with spatial dark solitons, they are experimentally formed in media characterized by a strong nonlinearityinduced change of the refractive index, e.g., alkali vapors (e.g., Rb), semiconductors (e.g., CdS), photorefractive crystals (e.g., SBN), photorefractive-photovoltaic crystals (e.g., $\mathrm{LiNbO}_{3}$ ), or polymers. In such cases, the function $F(I)$ in equation (1) is no longer a linear function of the light intensity $\left(I \equiv|\psi|^{2}\right)$ (i.e., the nonlinearity is of the non-Kerr type) and other models, such as the competing, saturable, and 

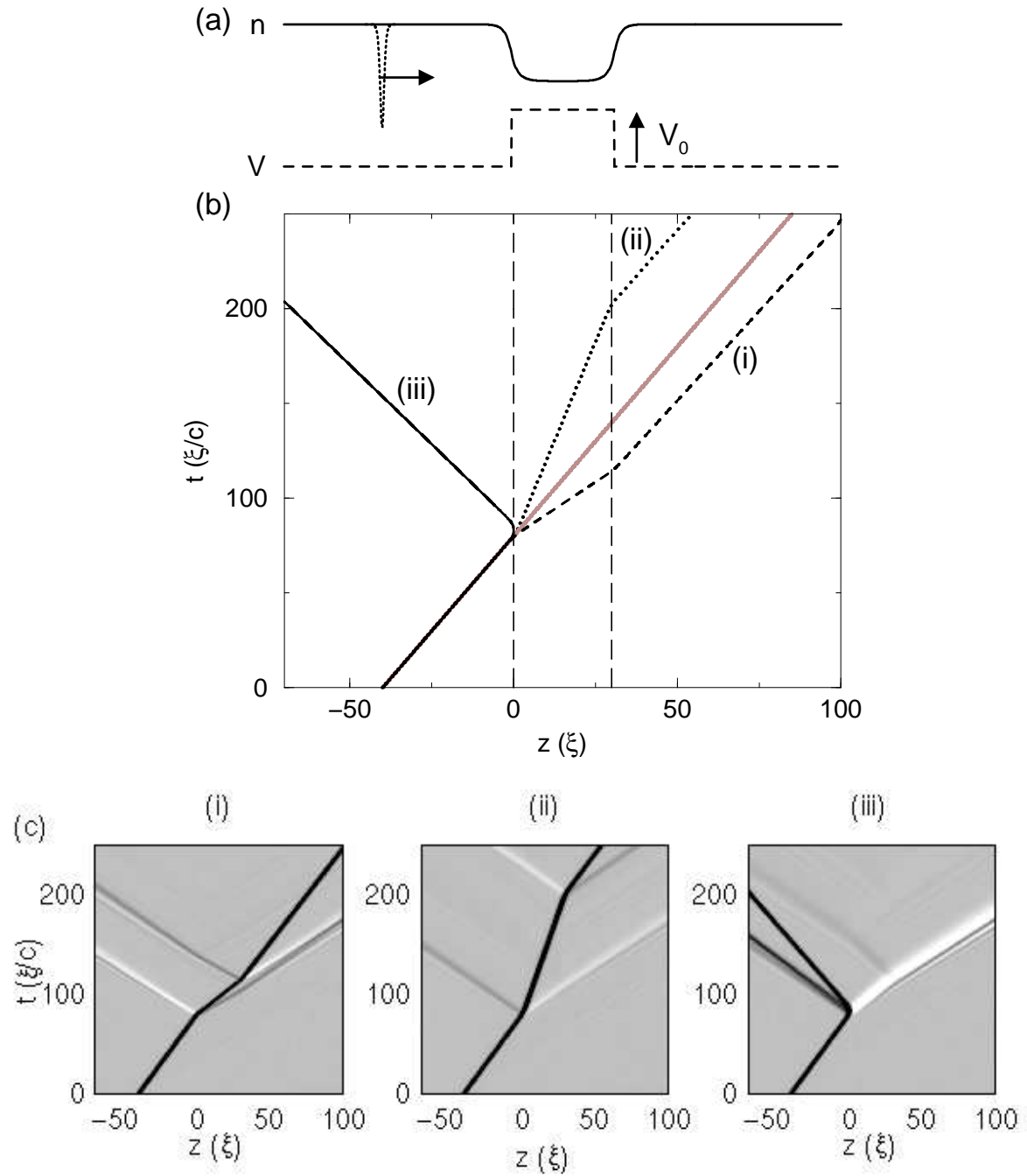

Figure 4. (a) Schematic of a dark soliton (dotted line) on a longitudinally homogeneous condensate background (solid line) incident on a finite potential step of height $V_{0}$ and length $30 \xi$ (dashed line). (b) Paths of a soliton of initial speed $v=0.5 c$ and position $z=-40 \xi$. (i) For $V_{0}<0\left(V_{0}=-0.5 \mu\right.$, dashed line), the soliton always transmits over the step; (ii) for positive and small enough $V_{0}$, the soliton transmits $\left(V_{0}=0.2 \mu\right.$, dotted line); and, (iii) for large and positive $V_{0}$ the soliton is reflected at the first interface $\left(V_{0}=0.5 \mu\right.$, solid black line). For ease of comparison, the soliton dynamics in the absence of the potential step is also included here (grey line). (c) Space-time carpet plots of the renormalised density for cases (i)-(iii) highlight the emission of counterpropagating sound waves (white/grey lines) whenever the soliton (black line) interacts with a boundary. These sound waves typically have an amplitude of a few percent of the peak density. Note that the emitted sound waves are also refracted at the interface (see, e.g. top left sound wave propagation of (c)(i)), albeit in a less pronounced manner, compared to the solitons. These and subsequent simulations are performed by the $1 \mathrm{D}$ GPE, in which the chemical potential is set by fixing the peak density to one. 


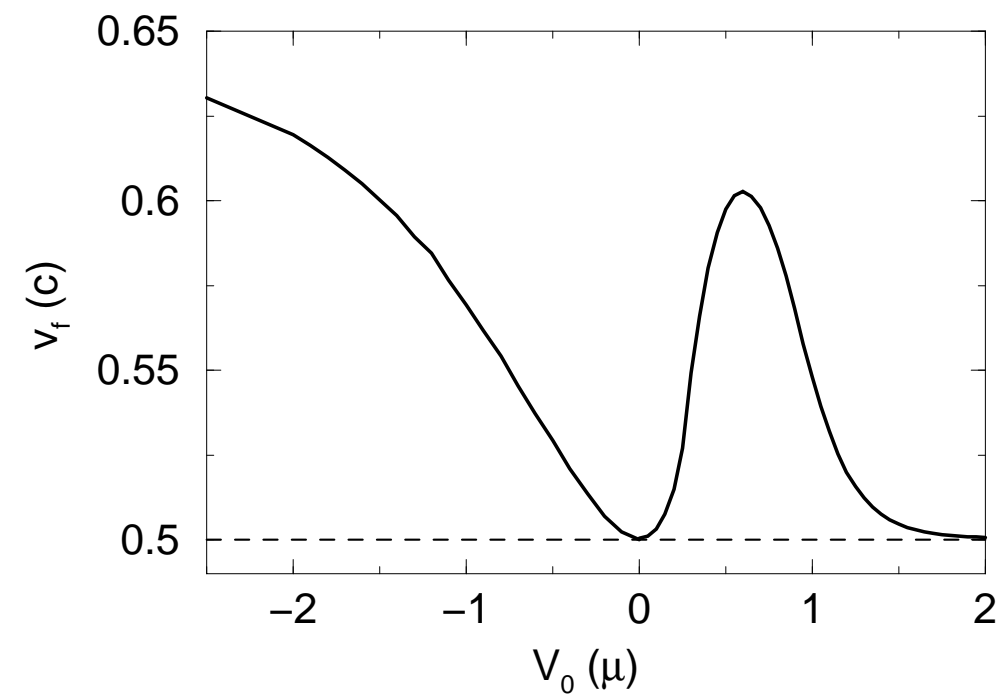

Figure 5. Final soliton velocity (solid line) after the interaction of a soliton, with initial speed $v=0.5 c$ (dashed line), with a potential step of height $V_{0}$ and length $L=30 \xi$. Finite step heights lead to an increase in the soliton speed due to the process of sound emission. For $V_{0}<0$ the soliton always transmits through the step region, with the emitted sound density, and therefore the final soliton velocity, increasing monotonically with $\left|V_{0}\right|$. Similarly, for $V_{0}>0$ up to some critical value, the soliton transmits, and the final velocity increases. Above this critical point, when the soliton becomes reflected, further increases of $V_{0}$ tend to reduce the velocity change, and for $V_{0}>2 \mu$ the soliton reflects elastically from the boundary with no change in its speed.

transiting nonlinearities are relevant [72]. In the framework of the nonintegrable (even in the absence of the inhomogeneous potential term) version of equation (1) with a general nonlinearity, the stability criterion for dark solitons was derived in [73 and, at the same time, the instability-induced dynamics of the dark solitons was investigated analytically and numerically in detail in 72. In the latter work, it has been demonstrated that the instability development is followed by emission of radiation, which propagates along the continuous-wave $(\mathrm{cw})$ pedestal inducing an effective dissipation to the dark soliton. Emission of radiation results in the acceleration of the dark soliton. In fact, these two quantities are intricately related, and the energy of the perturbed soliton decays by an acceleration squared law 72,

$$
\frac{\mathrm{d} E_{\mathrm{s}}}{\mathrm{d} t}=-L_{\mathrm{s}}(v, n)\left(\frac{\mathrm{d} v}{\mathrm{~d} t}\right)^{2} .
$$

The coefficient is given by

$$
\begin{aligned}
L_{\mathrm{s}}(v, n)=\frac{c}{c^{2}-v^{2}} & {\left[\frac{2 c^{2}}{n}\left(\frac{\partial N_{\mathrm{s}}}{\partial v}\right)^{2}\right.} \\
& \left.+2 v\left(\frac{\partial N_{\mathrm{s}}}{\partial v}\right)\left(\frac{\partial S_{\mathrm{s}}}{\partial v}\right)+\frac{n}{2}\left(\frac{\partial S_{\mathrm{s}}}{\partial v}\right)^{2}\right] .
\end{aligned}
$$



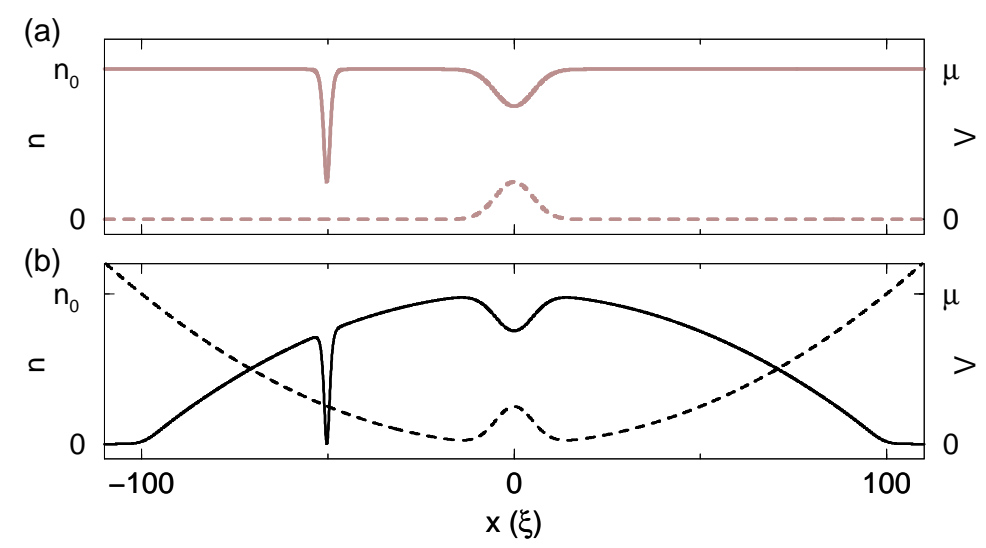

Figure 6. A dark soliton with initial position $z=-50 \xi$ incident on a gaussian bump of the form $0.25 \exp \left(-z^{2} / 50\right)$, for the cases of (a) homogeneous background density (optical fiber) and initial soliton speed $v=0.5 c$, and (b) longitudinal harmonic $\left(\omega_{z}=\sqrt{2} \times 10^{-2}(\mu / \hbar)\right.$ confinement (atomic traps) and an initially stationary soliton. In each case, the potential is marked by a solid line, and density by a dashed line.

and depends on the evolution of the total phase slip across the moving soliton $S_{\mathrm{s}}$, and the number of particles displaced by the soliton $N_{\mathrm{s}}=\int\left(n-|\psi|^{2}\right) \mathrm{d} z$ during this unstable motion. The quantities here have been expressed in terms of rates of change with $v$, which is one convenient way of formulating the problem, since $v$ is a continuously changing variable in this problem, and is directly related to the instability criterion for dark solitons [72].

\subsection{Dark Solitons in Quasi-1D Atomic BEC's}

The above mechanisms of decay do not apply directly to the atomic BEC case. In this case, the GPE is known to give, at sufficiently low temperatures, an accurate description of the dynamics of the system. Hence, in this case, decay will arise from other mechanisms, which could include quantum fluctuations [4], thermal damping from the uncondensed (normal) component of the system (i.e. the thermal cloud confined in the same trap) [61, or the effects of the longitudinal potential [49, 76]. Quantum fluctuations are expected to be more pronounced at small soliton speeds (nearly black solitons), and should not be that important for faster solitons. In addition to this, the thermal cloud is heavily suppressed at extremely low temperatures (like the ones in which soliton experiments have so far been performed), suggesting that this mechanism will only become significant for much higher temperatures. Hence, the longitudinal confinement is expected to be the key decay mechanism in the limit considered here. This effect has been considered in detail in [49, 70.

In this case, the soliton is dynamically unstable through its entire motion in the system, due to the fact that it constantly experiences a background density gradient. This is to be contrasted to the optical case, where instabilities due to modified nonlinearities only arise for a particular range of soliton speeds. The soliton oscillates in the harmonic trap [74, 75], and continuously emits energy in the form of sound waves [76]. However, the emitted sound energy remains confined within the 

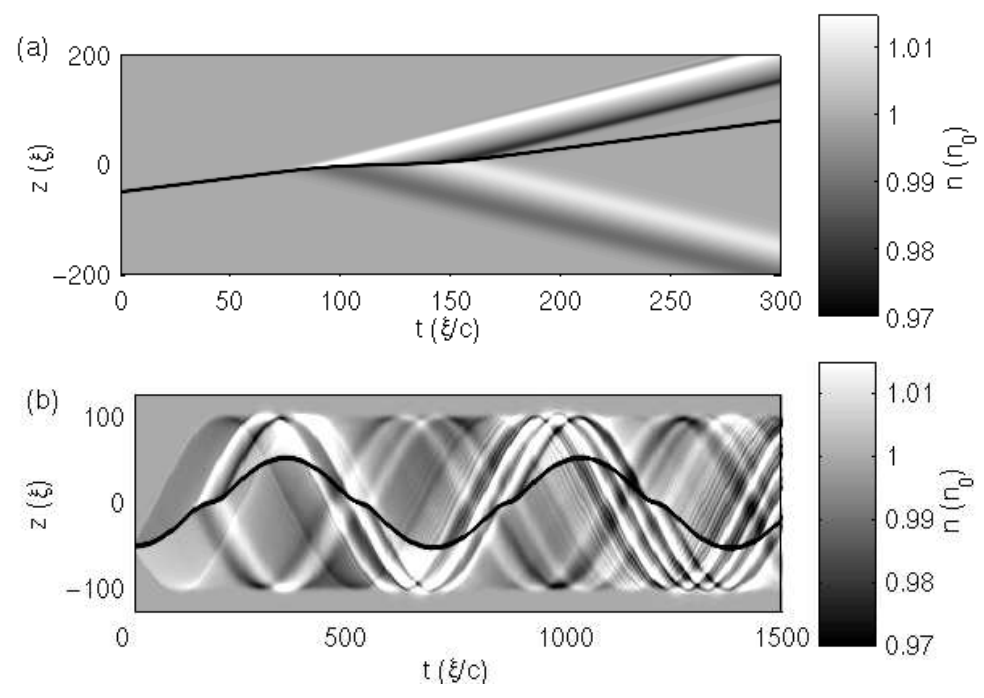

Figure 7. Renormalised density space-time plots depicting the long term evolution of a dark soliton, initially at $z=-50 \xi$, incident on a gaussian bump $0.25 \exp \left(-z^{2} / 50\right)$ in the cases of (a) a homogeneous system, and (b) a harmonic trap with longitudinal trapping $\omega_{z}=\sqrt{2} \times 10^{-2}(\mu / \hbar)$. In (a) the soliton, with initial speed $v=0.5 c$, emits counter-propagating sound pulses as it ascends and descends the bump. (b) The presence of the harmonic confinement induces periodic oscillations by the initially stationary solition, and hence repeated crossings over the bump. Although the actual emission process between these two cases is very similar (see figure 8), the harmonic potential traps the emitted sound, resulting in continuous soliton-sound interactions. The presence of the bump additionally dephases the emitted sound waves, resulting in the ultimate decay of the soliton after a sufficient number of passes over the bump. Note the different timescales and lengthscales in the two pictures.

same region, and hence the soliton continuously re-interacts with the emitted sound field [49. Nonetheless, one can observe the dissipation of the soliton energy by either (i) providing a suitable mechanism to damp off the emitted sound density, or (ii) causing the emitted sound waves to dephase, with both of these mechanisms leading to experimental proposals for controlling and measuring the effect of sound emission on soliton dynamics. In order to damp out the emitted sound, the soliton can be confined in a tight inner dimple trap, within a much weaker outer harmonic potential [49]. This situation can be readily realised by focusing an off-resonant laser beam within a magnetic harmonic trap. If the depth of the dimple trap is sufficiently shallow, the sound waves can escape to the outer trap, while the soliton remains confined in this region. In this limit, sound energy is removed for short enough timescales, until it bounces off the weaker outer trap and thus becomes forced to re-interact with the soliton in the inner dimple region. The second approach relies on soliton motion in a magnetic trap which is additionally perturbed by an optical lattice [77, 78] . In this case, the optical lattice can confine a soliton within a few lattice sites, with the sound (again for short enough times) escaping to further located lattice sites. Although the sound still reinteracts with the soliton, the presence of the periodic lattice potential dephases the emitted sound waves, and hence accelerates the decay of the soliton [77. 
To illustrate the effect of sound emission due to longitudinal background densities, consider the simple case of a dark soliton incident on a gaussian bump, as illustrated in figure 6. In the homogeneous case, the soliton will ascend and descend the bump, and emit two counter-propagating waves during its interaction with the bump (figure $7(\mathrm{a})$ ). These waves travel off to infinity and never reinteract with the soliton. The soliton speed is changed slightly due to the process of sound emission, as already discussed in section 3. However, the case of a gaussian bump (typically generated by a focused repulsive blue-detuned laser beam) in a system with longitudinal harmonic confinement is drastically different (figure $7(\mathrm{~b})$ ). The reason is that, in the absence of the bump, a dark soliton in a harmonic trap oscillates periodically in the trap, at a rate approximately equal to $\omega_{\text {trap }} / \sqrt{2}$ [46, 49, 71, 76, 79, 80, 81, where $\omega_{\text {trap }}$ is the longitudinal trap frequency. As the soliton oscillates in the trap, it continuously emits counter-propagating sound waves, due to the background density gradient which breaks the integrability of the system. However, the emitted sound remains confined within the trap, thus continually re-interacting with the soliton. This leads to a steadystate in which the soliton is stabilised against decay due to the complete reabsorption of the emitted sound 49, 76. The presence of the bump at the centre of the trap induces further dynamical instability in the soliton, with the additional sound emission being similar to that encountered in the homogeneous system (see figure 8 below). This additional decay mechanism leads to a more complicated carpet plot, shown in figure 7 (b), and eventually to the gradual decay of the dark soliton into a sound wave.

Focusing now on the initial part of the motion of the two systems, i.e. the interaction with the bump, figure 8 compares the homogeneous limit to harmonically confining traps, highlighting their similarities and differences. The sound emission due to the harmonic confinement means that the soliton arrives at the trap centre at different times in the trapped system compared to the homogeneous one. In order to compare the soliton dynamics, including the interaction of the soliton with the bump in the two cases, we hence find it convenient to shift the time axis of figure 8 with respect to that of figure 7 , such that $t=0$ corresponds to the time when both dark solitons corresponding to the homogeneous and the trapped cases reach the centre of the bump. Figure 8(a) shows the background densities experienced by the soliton in the two cases, with black corresponding to the harmonic case, and grey to the homogeneous limit. As the soliton starts ascending or descending the bump, it experiences a background density gradient which induces it to emit sound waves. Since the trap is quite shallow, the main acceleration experienced (solid lines in figure $8(\mathrm{~b})$ ) is due to the gaussian bump and is therefore similar in both cases. This is due to the fact that the background density gradient (as evident from figure 8(a)) is comparable in this region. The soliton starts decelerating, with the deceleration tailing off instantaneously to zero when the soliton is at the gaussian maximum, after which time the soliton accelerates down the bump. Far from the bump, there will, of course, be no sound emission occurring in the homogeneous case. This is, however, not the case for the harmonically confined system. The continuous harmonic density gradient induces sound emission during the entire motion of the system in the trap. Although this effect is instantaneously quite small (at most a few per cent of the background density), the cumulative effect can be significant.

The asymmetrically emitted sound contained within the 'soliton region' leads to an apparent deformation of the soliton profile. This deformation manifests itself as a shift of the soliton centre of mass $z_{c m}$ from the density minimum $z_{s}$, where the soliton 


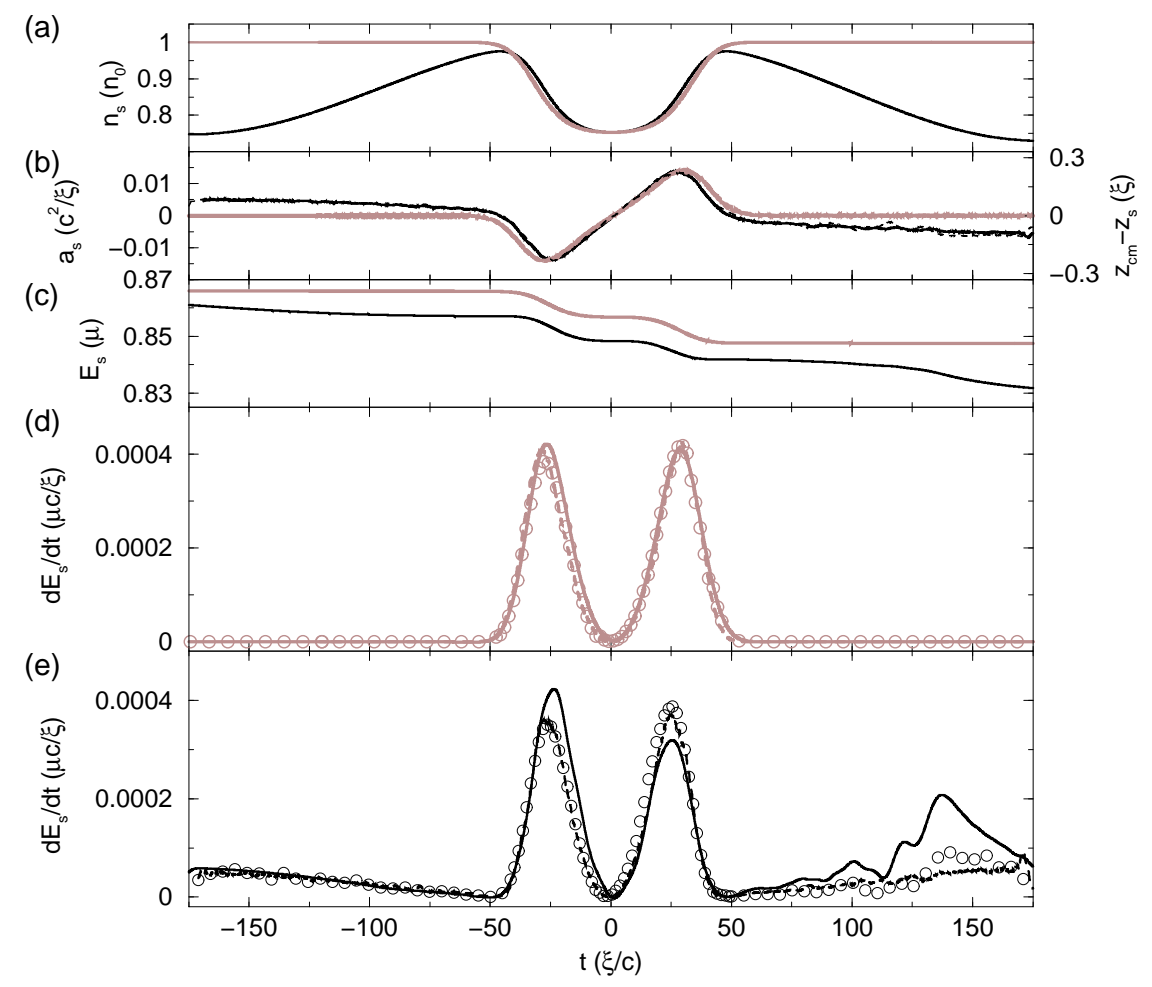

Figure 8. Dynamics of a dark soliton incident on a gaussian bump $0.25 \exp \left(-z^{2} / 50\right)$ in the cases of homogeneous (grey lines) and harmonic (black lines) confinement, corresponding to the cases of figures 6 and 7 . The time axes have been shifted such that the soliton reaches the centre of the bump at $t=0$. (a) Background density experienced by the moving soliton as a function of time. (b) Acceleration (solid lines, left axis) and deformation (open squares, right axis) of the soliton. (c) Soliton energy. (d) Rate of energy loss for the homogeneous system, as evaluated by the energy functional equation (7) (solid line), the acceleration squared law of equation (4) (open circles) and the deformation squared law of equation (8) with a constant coefficient $\kappa=0.0078\left(\mu c / \xi^{3}\right)$ (dashed line) determined by matching the maximum acceleration and deformation amplitudes in (b). (e) Same as (d) above, but for the harmonically confined case. The acceleration squared behaviour breaks down after about $t>75 \xi / c$, when the emitted sound starts reinteracting with the soliton, after having been reflected off the edge of the trap, leading to additional sound within the soliton region.

centre of mass is defined as

$$
z_{c m}=\frac{\int_{\mathrm{s}} z\left(|\psi|^{2}-n\right) \mathrm{d} z}{\int_{\mathrm{S}}\left(|\psi|^{2}-n\right) \mathrm{d} z}
$$

and the 'soliton region' $\mathrm{S}$ is conveniently taken to be $\left(z_{s} \pm 5 \xi\right)$. This deformation parameter, which is just another way of parameterising the instantaneously emitted sound density, is directly proportional to the acceleration, as indicated by the open squares and right axis of figure $8(\mathrm{~b})$.

The soliton energy is calculated numerically by integrating the GPE energy 
functional

$$
\varepsilon(\psi)=\frac{1}{2}|\nabla \psi|^{2}+V_{\text {ext }}|\psi|^{2}+\frac{1}{2}|\psi|^{4},
$$

across the soliton region $\mathrm{S}$ and subtracting the corresponding contribution from the time-independent background. This procedure cannot discriminate between soliton and sound energy present in the interval, and this will be shown to be intimately linked to the apparent soliton deformation. However, at least in the case of BECs, one could not discriminate between these two quantities in the same region.

The soliton energy (figure $8(\mathrm{c})$ ) is intimately related to the instantaneous acceleration and background density. As such, it will only decrease when the soliton accelerates, and this occurs stepwise for the homogeneous system, with instantaneously constant energy when the system is at the gaussian peak. The emission process can also be visualised by looking at the rate of energy loss due to sound emission. The picture in the two cases is again very similar, and is plotted respectively for homogeneous and trapped case in figure 8 (d) and (e). The zero emission rate at the centre of the gaussian bump is due to the locally homogeneous density and zero acceleration. The maximum magnitude of the sound emission is found to arise on the sides of the gaussian bump, when the background density gradient is at a maximum. The circles indicate numerical simulations based on equation (4), arising from nonlinear optics, while the dashed line is based on an emission law of the form

$$
\frac{\mathrm{d} E_{\mathrm{s}}}{\mathrm{d} t}=-\kappa\left(z_{c m}-z_{s}\right)^{2}
$$

with the value of $\kappa$ having been assigned such that it matches the emission. Although $\kappa$ will in general not be a constant, but rather a parameter dependent on the local soliton speed and background density gradient (similar to the coefficient of equation (5)), it is remarkable that we still find such good agreement with a constant coefficient [70]. We have performed detailed quantitative studies of the rate of sound emission in various geometries [49, 70, 77. Remarkably, we find that this rate is well described by an acceleration squared law, similar to that of the optical case. This may at first appear somewhat surprising, given the additional presence of the inhomogeneous potential in the atomic case, which was not included in the multiscale perturbative analysis leading to equation (4) [72 . However, in comparing our results, we have used soliton speeds obtained directly from the numerical simulations of the GPE, which hence take into account the modification on soliton speed and phase due to the harmonic confinement. In principle, one could rederive these equations in the presence of the harmonic confinement, although we do not consider this necessary here. To do this, one should combine the previously employed multiscale asymptotic techniques with boundary layer theory, as discussed in [76. At a time of $t \sim 75(\xi / c)$, we see a deviation of this law. This is due to the fact that the emitted sound has already travelled to the edge of the system (i.e. a point in the trapping potential beyond which it cannot ascend) and become reflected, thus returning towards the centre of the trap and reinteracting with the soliton. In the absence of the gaussian bump, such continuous interactions between soliton and emitted sound actually leads to the stabilisation of the soliton [49].

\section{Soliton Stabilisation}

In the case of optical dark solitons, inherent linear and nonlinear losses (the latter is related to two-photon absorption) of the host medium [7, 11] affect the $\mathrm{cw}$ background 
and render the dark soliton unstable. Generally speaking, losses can be compensated upon introducing a linear (intensity independent) gain. Nevertheless, although the latter stabilizes the background, the dark soliton remains unstable 82 and, as a result, dark solitons can be stabilized if the gain is nonlinear 83. Other stabilizing techniques include synchronized phase modulation 84, as well as phase-sensitive amplification and spectral filtering [85]. Additionally, robust parametrically driven dark solitons, immune from instabilities for all damping and forcing amplitudes have recently been reported 86 .

Generally speaking, parametric driving techniques may find application in the context of BECs, as a means to compensate the dissipative losses of dark solitons. In this case, an energy source is needed to compensate the continuous energy loss of the dark solitons due to the various decay mechanisms mentioned above. Work towards this direction is currently in progress. Importantly, even if this technique can not be applied in the BEC context, energy can be actually pumped into dark solitons via appropriate perturbations that may be realised by periodic potentials, somewhat analogously to the mechanism by which vortices acquire angular momentum from a laser stirrer. Soliton lifetimes can be noticeably enhanced in this way, and these results are presented elsewhere [87.

\section{Vortex Dynamics}

Although this paper deals with dark solitons, one should also mention a few words about other topological structures, such as vortices. In fact, vortices are not all that distinct from dark solitons, since, as outlined in section 2, a 3D dark soliton decays into vortex-like structures. These have already been observed in both optical and atomic systems.

An optical vortex represents a phase singularity in an optical beam, and is characterised by a helical wavefront and a dark core of zero intensity. The phase of the electric field integrated around the vortex core is an integer multiple of $2 \pi$. Optical vortices were first observed in 88 using a self-defocussing thermal nonlinearity and a phase mask which imposed an approximately helical phase structure of the vortex on the input beam. Another method for imprinting the above mentioned helical structure, based on a computer generated phase mask, was suggested in [89] and implemented in relevant experiments [90. On the other hand, to observe rotating optical vortices, a similar technique was used to create a pair of vortices with the same topological charge [91. Optical vortices may find applications as "optical tweezers" [92, while in a self-defocussing medium, an optical vortex acts as a dark waveguide 88.

The motion of an optical vortex filament due to both the interaction of other vortices and the presence of the background electric field has been shown to be strongly analogous to the hydrodynamic case [93. For example, a pair of identical optical vortex filaments in a gaussian beam have been observed to rotate about each other at a rate inversely proportional to the square of their separation [94. This result is exactly analogous to that for corotating vortices in an inviscid incompressible fluid. However, in a linear medium, the closely situated vortex cores rapidly expand into each other due to diffraction effects, while longer rotation angles can be achieved in self-defocussing media 95 .

In atomic BEC's, quantized vorticity can be excited by phase imprinting [27, 28], by stirring or rotating the system by magneto-optical techniques [96, or by the transverse decay of a dark solitary wave [97, and can appear in the form of single 

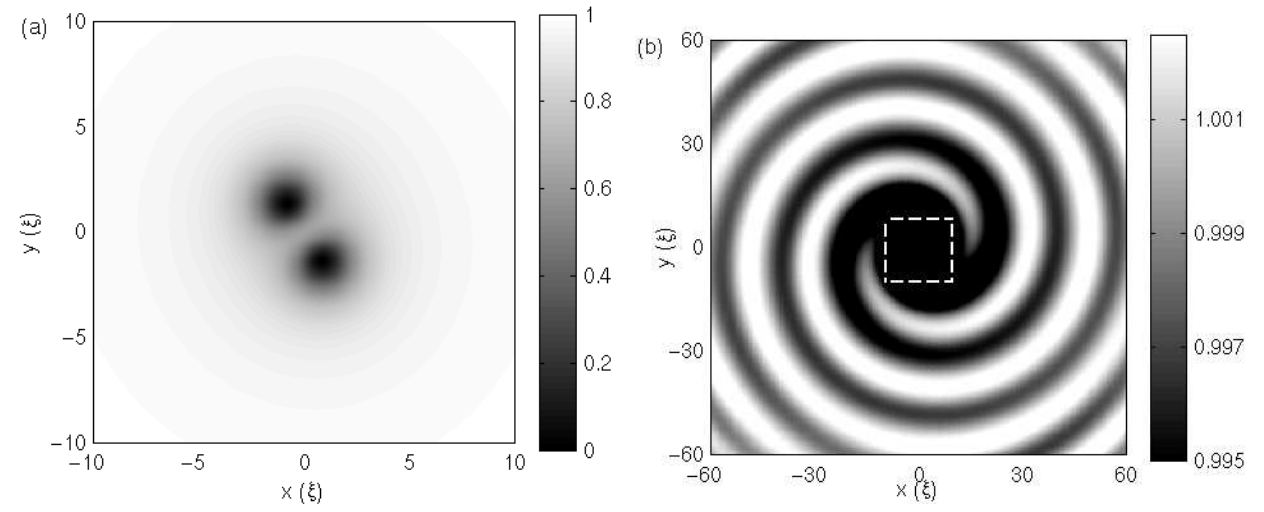

Figure 9. Two-dimensional density plots of a co-rotating vortex pair in a homogeneous system. The images are plotted on different greyscale contrast to highlight (a) the vortex pair and (b) the emitted sound pattern. Note that the sound profile is plotted on a much larger length-scale, with the region of image (a) corresponding to the dashed box in the centre of (b).

vortices, vortex lattices 98 and vortex rings. A single vortex tends to follow a line of constant potential and so an off-centered vortex will follow a circular trajectory around the trap centre 99, 100]. However, vortices are subject to a similar dynamical dissipation mechanism under acceleration as dark solitons, leading to the emission of sound 101]. If the emitted sound is suitably damped away the vortex will lose energy and spiral outwards [102, 103].

As an example of the dynamic instability of vortices, we consider here on the case of a closely positioned co-rotating vortex pair (two vortices of the same topological charge) in a homogeneous system. In analogy to the optical case outlined above, the vortices, separated by $2 r$, will rotate about their common axis at a rate proportional to $1 / r^{2}$, due to their mutual interaction. It has been shown analytically by Klyatskin 104105 that such a co-rotating pair will emit sound energy at a rate proportional to $1 / r^{6}$, and so slowly spiral apart. This result was obtained by looking at the far field emission pattern, and is hard to verify numerically. Instead, we illustrate the emission of sound in this process, by solving the GPE for a homogeneous two-dimensional system. For the co-rotating pair of figure 9 (a), the emitted sound is shown (on much larger scale) in figure $9(\mathrm{~b})$. The sound emitted by the rotating vortices creates a striking swirling pattern, which spreads outwards over time.

\section{Conclusions}

Many of the well-known nonlinear optics effects can now be readily observed in assemblies of ultracold Bose-Einstein condensed atomic gases. In the latter case, the wavefunction of the system obeys, at temperatures where the thermal component is suppressed, an equation similar to the equation for the electric field envelope in cubically nonlinear (Kerr) optical media. The nonlinearity in the atomic case arises from atomic interactions. This similarity leads to the observation of common phenomena, such as nonlinear wave mixing, bright and dark solitons and vortices, with this paper discussing at length the analogies between dark solitons in these two very 
different systems. In this context, we discussed the snake instability of dark solitons, arising from excitation of transverse modes in three-dimensional system. In the limit of tight transverse confinement, where such mechanisms are heavily suppressed, we showed that the leading decay mechanisms differ in the two systems. In real optical media used in experiments, one actually deals with a non-Kerr nonlinearity (e.g., a competing or a saturable nonlinearity), which renders the underlying system nonintegrable (even in the absence of the potential term, which may be used to describe a linear graded refractive index change). As a result, the dark solitons are unstable and their instability is accompanied by emission of radiation (or sound waves) that induce an effective dissipation to the dark solitons. In the limit of very low temperatures (where thermal effects can be ignored), the main instability in quasi-one-dimensional atomic condensates actually arises from sound emission along the line of motion, due to the harmonic confinement experienced (in stark contrast to the homogeneous optical media with a spatially-independent linear refractive index). In the absence of additional dephasing mechanisms (e.g. the additional presence of a gaussian bump, or a perturbing optical lattice), the continuous interaction between the oscillating dark soliton and the co-confined emitted sound actually leads to a steady-state preventing decay due to longitudinal confinement. Finally, vortex dynamics in both systems experience similar effects, with sound emission arising from the acceleration of vortices, due to either other topological charges, or background density gradients.

\section{Acknowledgments}

We acknowledge financial support from the UK EPSRC and the Special Research Account of the University of Athens.

\section{References}

[1] R.K. Dodd, J.C. Eilbeck, J.D. Gibbon, and H.C. Morris, Solitons and Nonlinear Wave Equations (Academic Press, London, 1982).

[2] J.L. Hammack and H. Segur, J. Fluid Mech. 65, 289 (1974).

[3] M. Peyrard (ed.), Molecular Excitations in Biomolecules (Springer-Verlag, New York, 1995).

[4] K.A. Naugol'nykh and L.A. Ostrovsky, Nonlinear Wave Processes in Acoustics (Cambridge University, Cambridge, 1998).

[5] H. Kim, R. Stenzel and A. Wong, Phys. Rev. Lett. 33, 886 (1974); H-H. Chen and C-S. Liu, Phys. Rev. Lett. 37, 693 (1976).

[6] A.M. Lomonosov, P. Hess and A.P. Mayer, Phys. Rev. Lett. 88, 076104 (2002).

[7] A. Hasegawa and Y. Kodama, Solitons in Optical Communications, (Oxford University Press, Oxford, UK, 1995); Yu. S. Kivshar and G.P. Agrawal, Optical Solitons: From Fibers to Photonic Crystals (Academic Press, Amsterdam, 2003).

[8] A. Mysyrowitz, Bose-Einstein Condensation, ed. by A. Griffin, D.W. Snoke and S. Stringari (Cambridge University Press, Cambridge, UK, 1995).

[9] J. Denschlag et al., Science 287, 97 (2000).

S. Burger et al., Phys. Rev. Lett. 83, 5198 (1999).

Z. Dutton, M. Budde, C. Slowe, and L.V. Hau, Science 293, 663 (2001).

[10] K.E. Strecher et al., Nature 417, 150 (2002). L. Khaykovich et al., Science 296, 1290 (2002).

[11] Yu.S. Kivshar and B. Luther-Davies, Phys. Rep. 278, 81-197 (1998).

[12] M. Nakazawa and K. Suzuki, Electron. Lett. 31, 1076 (1995); ibid 31, 1084 (1995).

[13] B. Luther-Davies and X. Yang, Opt. Lett. 17, 1775 (1992); S.Liu et al., Appl. Opt. 36, 8982 (1997).

[14] P. D. Miller, Phys. Rev. E 53, 4137 (1996).

[15] The relation between atomic and optical solitons has been discussed in a more mathematical manner in D. Schumayer and B. Apagyi, Phys. Rev. A 65, 053614 (2002). 
[16] F. Dalfovo et al. Rev. Mod. Phys. 71, 463 (1999).

[17] S. Raghavan and G.P. Agrawal, Opt. Commun. 180, 377 (2000).

[18] V.E. Zakharov and A.B. Shabat, Zh. Eksp. Teor. Fiz. 64, 1627 (1973) [Sov. Phys. JETP 37, 823 (1973)].

[19] A. Hasegawa and F. Tappert, Appl. Phys. Lett. 23, 171 (1973).

[20] Ph. Emplit et al., Opt. Commun. 62, 374 (1987).

[21] D. Krökel et al., Phys. Rev. Lett. 60, 29 (1988).

[22] A.M. Weiner et al., Phys. Rev. Lett. 61, 2445 (1988).

[23] J.A.R. Williams et al., Opt. Commun. 112, 333 (1994); D.J. Richardson et al., Electron. Lett. 30, 1326 (1994).

[24] G.A. Swartzlander et al., Phys. Rev. Lett. 66, 1583 (1991).

[25] G.R. Allan et al., Opt. Lett. 16, 156 (1991).

[26] G. Duree et al., Phys. Rev. Lett. 74, 1978 (1995).

[27] M.R. Matthews et al. Phys. Rev. Lett. 83, 2498 (1999).

[28] J.E. Williams and M.J. Holland. Nature 568, 401 (1999).

[29] L. Dobrek et al., Phys. Rev. A 60, R3381 (1999).

[30] K. Bongs et al., J. Opt. B: Quantum Semiclass. Opt. 5, S124 (2003).

[31] R. Dum, J.I. Cirac, M. Lewenstein and P. Zoller, Phys. Rev. Lett. 80, 2972 (1998).

[32] L.D. Carr et al., Phys. Rev. A 63, 051601 (2001).

[33] S. Burger et al., Phys. Rev. A 65, 043611 (2002).

[34] S.A. Gredeskul and Yu.S. Kivshar, Phys. Rev. Lett. 62, 977 (1989); S.A. Gredeskul, Yu.S. Kivshar, and M.V. Yanovskaya, Phys. Rev. A 41, 3994 (1990).

[35] D.S. Petrov, G.V. Shlyapnikov and J.T.M. Walraven, Phys. Rev. Lett. 85, 3745 (2000).

[36] U. Al Khawaja, J.O. Andersen, N.P. Proukakis and H.T.C. Stoof, Phys. Rev. A 66, 013615 (2002).

[37] A. Goerlitz et al., Phys. Rev. Lett. 87, 130402 (2001).

[38] M. Olshanii, Phys. Rev. Lett. 81, 938 (1998).

[39] W. Haensel et al., Nature 413, 498 (2001).

[40] F. Schreck et al., Phys. Rev. Lett. 87, 080403 (2001).

[41] H. Ott et al., Phys. Rev. Lett. 87, 230401 (2001).

[42] M.P.A. Jones, C.J. Vale, D. Sahagun, B.V. Hall and E.A. Hinds, Phys. Rev. Lett. 91, 080401 (2003).

[43] A.D. Jackson, G.M. Kavoulakis and C.J. Pethick, Phys. Rev. A 58, 2417 (1998).

[44] A.E. Muryshev, H.B. van Linden van den Heuvell, and G.V. Shlyapnikov, Phys. Rev. A 60, R2665 (1999).

[45] E.M. Lifshitz and L.P. Pitaevskii, Statistical Physics Part 2 (Butterworth, Heinemann, Oxford, 1998).

[46] P. O. Fedichev, A. E. Muryshev, and G. V. Shlyapnikov, Phys. Rev. A 60, 3220 (1999).

[47] E.B. Kolomeisky et al., Phys. Rev. Lett. 85, 1146 (2000).

[48] J. Dziarmaga, Z. P. Karkuszewski, and K. Sacha, J. Phys. B 36, 1217 (2003).

[49] N.G. Parker, N.P. Proukakis, M. Leadbeater, and C.S. Adams, Phys. Rev. Lett. 90, 220401 (2003).

[50] E.A. Kuznetsov and S.K. Turitsyn, Zh. Eksp. Teor. Fiz. 94, 119 (1988) [Sov. Phys. JETP 67, 1583 (1988)]; E.A. Kuznetsov and J.J. Rasmussen, Phys. Rev. E 51, 4477 (1995); D.E. Pelinovsky, Yu.A. Stepanyantz and Yu.S. Kivshar, Phys. Rev. E 51, 5016 (1995).

[51] D.R. Andersen et al., Opt. Lett. 15, 783 (1990).

[52] V. Tikhonenko et al., Opt. Lett. 21, 1129 (1996);

[53] A.V. Mamaev, M. Saffman and A.A. Zozulya, Phys. Rev. Lett. 76, 2262 (1996); A.V. Mamaev et al., Phys. Rev. A 54, 870 (1996).

[54] Yu.S. Kivshar and D.E. Pelinovsky, Phys. Rep. 331, 117 (2000).

[55] B.P. Anderson et al., Phys. Rev. Lett. 86, 2926 (2001).

[56] D.L. Feder et al., Phys. Rev. A 62, 053606 (2000).

[57] J. Brand and W.P. Reinhardt, Phys. Rev. A 65, 043612 (2002).

[58] Yu.S. Kivshar and X. Yang, Phys. Rev. E 50, R40 (1994).

[59] A. Dreischuh et al., Phys. Rev. E 66, 066611 (2002).

[60] G. Theocharis et al., Phys. Rev. Lett. 90, 120403 (2003).

[61] A. Muryshev et al, Phys. Rev. Lett. 89, 110401 (2002).

[62] S. Komineas and N. Papanikolaou, Phys. Rev. A 68, 043617 (2003).

[63] J.V. Moloney and A.C. Newell, Physica D 44, 1 (1990).

[64] A.B. Aceves, J.V. Moloney and A.C. Newell, Phys. Lett A 129, 231 (1998); J. Opt. Soc. Am. B 5, 559 (1988); Phys. Rev. A 39, 1809 (1989); ibid 39, 1828 (1989). 
Dark solitons in atomic condensates and optical systems

[65] Yu.S. Kivshar and B.A. Malomed, Rev. Mod. Phys. 61, 763 (1989).

[66] Yu.S. Kivshar, A.M. Kosevich and O.A. Chubykalo, Phys. Rev. A 41, 1677 (1990).

[67] Yu.S. Kivshar and M.L. Quiroga-Teixeiro, Phys. Rev. A 48, 4750 (1993).

[68] Yu.S. Kivshar, A.M. Kosevich and O.A. Chubykalo, Phys. Lett. A 125, 35 (1987).

[69] Yu.S. Kivshar, A.M. Kosevich and O.A. Chubykalo, Zh. Eksp. Teor. Fiz. 93, 968 (1987) [Sov. Phys. JETP 66, 545 (1987)].

[70] N. G. Parker, N. P. Proukakis, M. Leadbeater, and C. S. Adams, J. Phys. B 36, 2891 (2003).

[71] D.J. Frantzeskakis, G. Theocharis, F.K. Diakonos, P. Schmelcher, and Yu.S. Kivshar, Phys. Rev. A 66, 053608 (2002).

[72] D.E. Pelinovsky, Y.S. Kivshar. and V.V. Afanasjev, Phys. Rev. E 54, 2015 (1996).

[73] I.V. Barashenkov, Phys. Rev. Lett. 77, 1193 (1996).

[74] W.P. Reinhardt and C.W. Clark, J. Phys. B: At. Mol. Opt. Phys. 30, L785 (1997).

[75] S.A. Morgan, R.J. Ballagh and K. Burnett, Phys. Rev. A 55, 4338 (1997).

[76] T. Busch and J. R. Anglin, Phys. Rev. Lett. 84, 2298 (1999).

[77] N. G. Parker, N. P. Proukakis, C. F. Barenghi and C. S. Adams, J. Phys. B 37 (In Press, 2004).

[78] G. Theocharis, D.J. Frantzeskakis, P.G. Kevrekidis, R. Carretero-Gonzalez, and B.A. Malomed, subm. to Mathematics and Computers in Simulation (2003).

[79] G. Huang, J. Szeftel, and S. Zhu, Phys. Rev. A 65, 053605 (2002).

[80] N.P. Proukakis, N.G. Parker, C.F. Barenghi and C.S. Adams, Las. Phys. 14, 284 (2004).

[81] V.A. Brazhnyi and V.V. Konotop, Phys. Rev. A 68, 043613 (2003).

[82] Yu.S. Kivshar and X. Yang, Phys. Rev. E 49, 1657 (1994).

[83] T. Ikeda, M. Matsumoto, and A. Hasegawa, Opt. Lett. 20, 1113 (1995); J. Opt. Soc. Am. B 14, 136 (1997); X.J. Chen and Z.D. Chen, IEEE J. Quantum Electron. 34, 1308 (1998).

[84] A. Maruta and Y. Kodama, Opt. Lett. 20, 1752 (1995).

[85] A.D. Kim, W.L. Kath and C.G. Goedde, Opt. Lett. 21, 465 (1996).

[86] I.V. Barashenkov, S.R. Woodford, and E.V. Zemlyanaya, Phys. Rev. Lett. 90, 054103 (2003).

[87] N.P. Proukakis, N.G. Parker, C.F. Barenghi and C.S. Adams, Preprint (2004).

[88] G. A. Swartzlander, Jr. and C. T. Law, Phys. Rev. Lett. 69, 2503 (1992).

[89] N.R. Heckenberg et al., Opt. Lett. 17, 221 (1992).

[90] V. Tikhonenko, J. Christou, and B. Luther-Davies, J. Opt. Soc. Am. B 12, 2046 (1995); Phys. Rev. Lett. 76, 2698 (1996).

[91] B. Luther-Davies, R. Powles, and V. Tikhonenko, Opt. Lett. 19, 1816 (1994).

[92] N. B. Simpson, L. Allen, and M. J. Padgett, J. Mod. Opt. 43, 2485 (1996).

[93] D. Rozas, C. T. Law, and G. A. Swartlander, Jr., J. Opt. Soc. Am. B 14, 3054 (1997).

[94] D. Rozas, Z. S. Sacks, and G. A. Swartzlander, Jr., Phys. Rev. Lett. 79, 3399 (1997).

[95] D. Rozas and G. A. Swartzlander, Jr., Opt. Lett. 25, 126 (2000).

[96] C. Raman et al., Phys. Rev. Lett. 832498 (1999).

K.W. Madison et al., Phys. Rev. Lett. 84, 806 (2000).

E. Hodby et al., Phys. Rev. Lett. 88, 010405 (2002).

[97] B.P. Anderson et al., Phys. Rev. Lett. 86, 2926 (2001).

[98] J.R. Abo-Shaeer et al., Science 292, 476 (2001).

[99] B.P. Anderson et al. Phys. Rev. Lett. 85, 2857 (2000).

[100] A. Fetter and A. Svidzinsky, J. Phys. Condens. Matter 13, R135 (2001).

[101] E. Lundh and P. Ao, Phys. Rev. A 61, 063612 (2000).

[102] P.G. Kevrekidis, R. Carretero-Gonzalez, G. Theocharis, D.J. Frantzeskakis and B.A. Malomed, J. Phys. B: At. Mol. Opt. Phys. 36, 3467 (2003).

[103] N.G. Parker, N.P. Proukakis, C.F. Barenghi and C.S. Adams, cond-mat/0312520 (2003).

[104] V. I. Klyatskin, Izn. AN. SSSR Mekh. Zh. Gaz 6, 87 (1966).

[105] L.M. Pismen, Vortices in nonlinear fields (Clarendon Press, Oxford, 1999). 